\title{
Adsorption Thermodynamics in the Framework of the Modified Associate Formalism
}

\author{
D. N. Saulov ${ }^{1}$, V. Rudolph ${ }^{2}$ and A. Y. Klimenko,* \\ ${ }^{I}$ School of Mechanical and Mining Engineering, The University of Queensland, Australia \\ ${ }^{2}$ School of Chemical Engineering, The University of Queensland, Australia
}

\begin{abstract}
A new thermodynamic model of adsorption of gas mixtures on microporous adsorbents is suggested. The model is based on Vacancy Solution Theory, while the recently proposed Modified Associate Formalism is used for adsorbed phase. The proposed model allows prediction of co-adsorption isotherms based on fitting those for pure gases.
\end{abstract}

Keywords: Adsorption, vacancy solution theory, modified associate formalism.

\section{INTRODUCTION}

Underground storage of carbon dioxide is one of the prominent options for reducing its release in the atmosphere in order to tackle the problem of climate change. $\mathrm{CO}_{2}$ injection into coal seams also enhances the recovery of Coal Bed Methane (CBM) [1]. This, in turn, reduces the overall cost of carbon storage and maximises resource utilization. Since gas species are present in coal mainly in the adsorbed state, accurate modeling of adsorption of gas mixtures on coal is of particular importance for the development of advanced technologies of both $\mathrm{CO}_{2}$ sequestration and enhanced CBM recovery.

As pointed out, for example, by Koter and Terzyk [2], the thermodynamics of adsorption can be treated in two alternative approaches. In the first one, the adsorbed phase is treated as 2-dimensional surface phase, which is characterised by the surface area and the spreading pressure. The second approach involves treatment of the adsorbed phase as a solution of adsorbates and vacancies.

Myers [3] discussed disadvantages of the conventional 2D approach for modelling the thermodynamics of adsorption in porous materials. As pointed out by the author, the 2-D approach requires several assumption of questionable validity, such as inert adsorbent, perfect gas, negligible volume of the adsorbed phase and so on. Moreover, neither surface area nor spreading pressure can be measured experimentally or calculated theoretically for materials with complex pore structures. According to Myers [3], the 2-D approach is not justified for modelling the thermodynamics of adsorption in porous materials.

The second approach pioneered by Bering et al. $[4,5]$ is also known as Vacancy Solution Theory (VST). VST has

*Address correspondence to this author at the School of Mechanical and Mining Engineering, The University of Queensland St. Lucia, QLD 4072, Australia; Tel: +61-(0)7+3365-3670; E-mail: a.klimenko@uq.edu.au been successfully used in many studies to describe adsorption processes on various adsorbents (including those with highly complicated pore structures) for wide ranges of temperature and pressure. It has also been demonstrated [2] that several currently used adsorption models (adsorption isotherms) can be derived from VST. In this paper, we follow VST, while utilising the recently suggested Modified Associate Formalism (MAF) [6] to model the adsorbed phase.

\section{MODIFIED ASSOCIATE FORMALISM}

It is well understood that the quality of the isotherm derived from VST drastically depends on the quality of the solution model used to describe the adsorbed phase. MAF is capable of taking into account strong and complex (multi-particle) interactions between mixing species in a thermodynamically and mathematically consistent way. At the same time, MAF correctly reduces to the ideal solution model in the case of zero Gibbs free energy of mixing.

Advantages of MAF over other models currently used to describe solution phases with strong interactions between mixing particles have been demonstrated [7]. MAF utilizes the expression for configurational entropy which is thermodynamically correct and free from the entropy paradox. The adjustable parameters used have clear physical meaning, while providing sufficient flexibility for fitting experimental data. The model is applicable to the entire compositional range, which is of particular importance for modeling high loading regimes. Ability to model multicomponent adsorption is an integral part of the proposed model. The advantages of MAF provide a potential for development of the adsorption model of high quality. In this section we outline the formalism. The detail description of the model for an arbitrary number of the solution component and an arbitrary size of the associates is presented in the previous work [6].

Consider a binary solution $A-B$ with 3-particles associates. The particle sites within an associate are 
distinguishable and can be numbered. If the $1^{\text {st }}$ and $2^{\text {nd }}$ sites in an associate are both occupied by $A$-particles, while the $3^{\text {rd }}$ one is occupied by a $B$-particle, such an associate is said to be of type $[A A B]$. Other types of associates are defined similarly. Thus, there are $2^{3}=8$ different types of associates. Note that all these types should be taken into account in calculating the configurational entropy. The associates, which consist of both $A$ and $B$ particles, are referred to as mixed associates, while $[A A A]$ and $[B B B]$ associates are said to be pure associates.

The molar Gibbs free energy of the solution is given by

$G=\sum_{i, j, k=A, B} n_{[i j k]}\left(g_{[i j k]}+R T \ln x_{[i j k]}\right)$.

Here, $g_{[i j k]}, n_{[i j k]}$ and $x_{[i j k]}$ are the molar Gibbs free energy, the mole number and the molar fraction of [ijk]associates, respectively. In one mole of pure $A$ there are $1 / 3$ moles of $[A A A]$-associates. Hence, $g_{[A A A]}=3 g_{A}$, where $g_{A}$ is the molar Gibbs free energy of the pure solution component $A$. Similarly, $g_{[B B B]}=3 g_{B}$.

The molar Gibbs free energies $g_{[i j k]}$ of mixed associates are adjustable parameters of the model, which can depend on the temperature $T$ as

$g_{[i j k]}=h_{[i j k]}-T s_{[i j k]}$.

Instead of $g_{[A A B]}$, for example, one can use the Gibbs free energy change $\Delta g_{[A A B]}$ on forming [AAB]-associates from pure associates according to the following reaction as the adjustable parameter of the model.

$\frac{2}{3}[A A A]+\frac{1}{3}[B B B] \rightleftarrows[A A B]$.

$\Delta g_{[A A B]}$ is given by

$\Delta g_{[A A B]}=g_{[A A B]}-\frac{2}{3} g_{[A A A]}-\frac{1}{3} g_{[B B B]}$.

The other $\Delta g_{[i j k]}$ of mixed associates are defined similarly.

At equilibrium, the molar fractions $x_{[i j k]}$ of mixed associates are given by

$\frac{x_{[i j k]}}{\left(x_{[A A A]}\right)^{\alpha_{[i j k]^{3}}}\left(x_{[B B B]}\right)^{\beta_{[i j k]} / 3}}=\exp \left(-\frac{\Delta g_{[i j k]}}{R T}\right)$.

Here, $\alpha_{[i j k]}$ and $\beta_{[i j k]}$ are the numbers of $A$ and $B$ particles in the $[i j k]$-associate. For example, $\alpha_{[A A B]}=2$ and $\beta_{[A A B]}=1$.

The chemical potential $\mu_{A}$ of the solution component $A$ is expressed as

$\mu_{A}=\frac{g_{[A A A]}+R T \ln \left(x_{[A A A]}\right)}{3}$.

The chemical potential $\mu_{B}$ is calculated similarly.

\section{EQUATIONS FOR CALCULATING THE ADSORP- TION ISOTHERM}

In this section, we present the equations for calculating the adsorption isotherm for pure gas. Extension of these equations for gas mixtures is straightforward. The adsorbed phase is treated as the solution of adsorbate $A$ and vacancies $B$. Following the technique used by Ding and Bhatia $[8,9]$, the adsorption of a molecule of $A$ is described by the reaction

$A^{\mathrm{g}}+B^{\mathrm{a}} \leftrightarrows A^{\mathrm{a}}$

Here and later in the paper, the superscripts "a" and "g" indicate adsorbed and gas phase, respectively. We also assume that one vacancy is consumed per one molecule of A.

Let $\Delta G$ be the Gibbs free energy change in the reaction (7). The equilibrium condition is then given by

$\frac{\partial \Delta G}{\partial n}=\mu_{A}^{\mathrm{a}}-\mu_{B}^{\mathrm{a}}-\mu_{A}^{\mathrm{g}}=0$

where $\mu_{A}^{\mathrm{g}}$ and $\mu_{A}^{\mathrm{a}}$ are the chemical potential of $A$ in gas and adsorbed phases, $\mu_{B}^{\mathrm{a}}$ is the chemical potential of vacancies and $n$ is the mole number of $A$. The chemical potential $\mu_{A}^{\mathrm{g}}$ in the gas phase is assumed to be a known function of temperature $T$ and pressure $p$.

Now consider one mole of 3-particle associates at equilibrium with the gas $A$. Using Eq. (6), the equilibrium condition (8) takes the form.

$\ln \left(x_{[A A A]}\right)-\ln \left(x_{[B B B]}\right)=\frac{3 \mu_{A}^{\mathrm{g}}+g_{[B B B]}-g_{[A A A]}}{R T}$.

The molar fractions $x_{[i j k]}$ are subject to the mass balance constraint

$\sum_{i, j, k=A, B} x_{[i j k]}=1$.

The molar fractions $x_{[i j k]}$ with $[i j k] \neq[A A A],[B B B]$ are given by Eq. (5). Thus, Eqs. (5), (9) and (10) form a closed set of algebraic equations for calculating the equilibrium composition of the adsorbed phase.

Generally, it is impossible to obtain explicit analytical expressions for equilibrium values of $x_{[i j k]}$. This disadvantage, however, is endurable, since an effective algorithm for calculating equilibrium composition of the adsorbed phase can be implemented in a straightforward way. For the purposes of this paper, for example, the equilibrium concentrations have been calculated by solving the Eqs. (5), (9) and (10) numerically, using the embedded Microsoft Excel tool called "Solver".

Let $N_{\mathrm{m}}$ be the mole number of associates per unit mass of adsorbent. Recalling that $\alpha_{[i j k]}$ are the numbers of $A$ particles in the $[i j k]$-associate, the mole number $n_{\mathrm{m}}$ of adsorbed $A$-particles per unit mass of adsorbent is calculated as 
$n_{\mathrm{m}}=N_{\mathrm{m}} \sum_{i, j, k=A, B} \alpha_{[j k]} x_{[i j k]}$

Note that $N_{\mathrm{m}}$, which depends on many factors, including porosity and pore structure of the adsorbent, is the adjustable parameter of the model.

\section{EXAMPLE APPLICATION}

Goetz et al. [10] measured adsorption of pure methane and carbon dioxide on activated carbon for pressure up to 3.5 $\mathrm{MPa}$ and their mixture for $0.1,0.5$ and $1 \mathrm{MPa}$. Adsorption of pure gases was modelled using the Dubinin-Astakhov (DA) equation [11]. To model the adsorption of gas mixture the authors followed the approach by Lavanchy et al. [12] that combines the Ideal Adsorbed Solution Theory (IAST) [13] with the DA equation. The reported experimental results are used to demonstrate applicability of the model suggested in the present study and to compare it with the approach used in Ref. [10].

Since the pressure in question is low or moderate, the ideal gas approximation is used. Thus, the chemical potentials $\mu_{i}^{\mathrm{g}}$ are given by

$\mu_{i}^{\mathrm{g}}=g_{i}^{\circ}+R T \ln \left(\frac{p_{i}}{p^{\circ}}\right)$,

where $g_{i}^{\circ}$ are molar Gibbs free energies of pure gases at the reference pressure $p^{\circ}=1$ bar and $p_{i}$ are the partial pressures of gas species. For $T=298 \mathrm{~K}, g_{i}^{\circ}$ are taken from Ref. [14]. For $T=273 \mathrm{~K}, g_{i}^{\circ}$ are estimated using the approximation of constant heat capacity.

The adsorbed phase is modelled using 3-particle associates. For the sake of simplicity, it is assumed that the associates of the same "chemical composition" have equal Gibbs free energy. For example,

$g_{[C C V]}=g_{[C V C]}=g_{[V C C]} \equiv g_{C_{2} V}$,

$g_{[M M V]}=g_{[M V M]}=g_{[V M M]} \equiv g_{M_{2} V}$.
Here and later in the paper, " $C$ " and " $M$ " denote molecules of carbon dioxide and methane, while vacancies are denoted by " $V$ ".

Table 1. Gibbs Free Energy Changes on Forming Mixed Associate from Pure Associate

\begin{tabular}{|l|l|}
\hline Associate & $\Delta g[\mathbf{k J} / \mathbf{m o l}]$ \\
\hline \hline$V_{2} C$ & -10.5 \\
\hline$V C_{2}$ & -17.0 \\
\hline$V_{2} M$ & -6.6 \\
\hline$V M_{2}$ & -6.5 \\
\hline$C_{2} M$ & 0 \\
\hline$C M_{2}$ & 0 \\
\hline$C M V$ & 0 \\
\hline
\end{tabular}

It is found that the temperature dependence of the Gibbs free energies of the associates (see Eq. (2)) is not required for accurate fitting of the experimental data in question. Such a dependence, however, can be useful for more complex cases, where wider temperature ranges are involved.

Since modelling of heat effects is beyond the scope of the present study, the Gibbs free energies of associates are taken in the reference state, where $g_{V_{3}}=0$. In this reference state, the Gibbs free energies of the associates and the value for $N_{\text {m }}$ where simultaneously chosen by fitting the experimental data. The values for pure associates where selected as follows: $g_{C_{3}}=-1330.8 \mathrm{~kJ} / \mathrm{mol}$ and $g_{M_{3}}=-352.5 \mathrm{~kJ} / \mathrm{mol}$. The values of Gibbs free energy changes on forming mixed associates from pure associates are given in Table $\mathbf{1}$, while the value for $N_{\mathrm{m}}$ is selected to be $18 \mathrm{~mol} / \mathrm{kg}$. Measured and calculated adsorption isotherms for pure methane and carbon

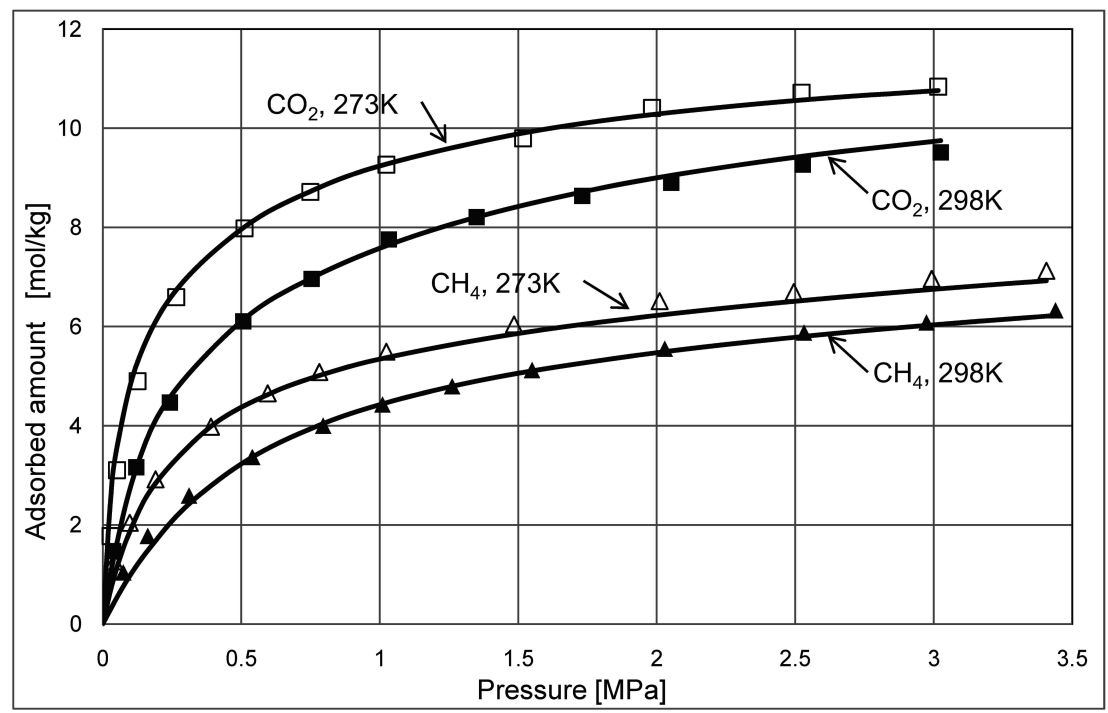

Fig. (1). Adsorption isotherms on activated carbon for pure $\mathrm{CO}_{2}$ and $\mathrm{CH}_{4}$. Symbols represent experimental data taken from Ref. [10] ( $\square-\mathrm{CO}_{2}$ at $273 \mathrm{~K}, \boldsymbol{-}-\mathrm{CO}_{2}$ at $298 \mathrm{~K}, \Delta-\mathrm{CH}_{4}$ at $273 \mathrm{~K}, \boldsymbol{\Delta}-\mathrm{CH}_{4}$ at $298 \mathrm{~K}$ ), while solid lines show values calculated by the model. See also Fig. (2) in Ref. [10]. 
dioxide are given in Fig. (1). Modelling of co-adsorption is summarised in Figs. $(\mathbf{2}, \mathbf{4})$.

Note that $V_{2} C$ and $V C_{2}$ are used to fit the experimental data for pure $\mathrm{CO}_{2}$, while $V_{2} M$ and $V M_{2}$ are used for pure $\mathrm{CH}_{4}$. The other adjustable parameters $\left(C_{2} M, C M_{2}\right.$ and $C M V$ ) can be used for the $\mathrm{CO}_{2} / \mathrm{CH}_{4}$ mixture. In the present study, however, the values of these parameters have been kept zero (see Table 1). It is done in order to test the ability of the model to predict the adsorption behaviour of a gas mixture based on fitting the experimental data for pure gases.
Comparing Figs. (1-4) in this paper with Figs. (2,3,6 and 7) in Ref. [10], respectively, one verifies that the suggested model is at least of the same quality as the approach used by Goetz et al. At the same time, the present model provides better fitting of the co-adsorption experimental data at 0.5 and $1 \mathrm{MPa}$ for the $\mathrm{CO}_{2} / \mathrm{CH}_{4}$ mixtures with high $\mathrm{CO}_{2}$ content.

\section{CONCLUSION}

New model for treating the adsorption thermodynamics of gas mixtures is suggested. The model is based on VST, while MAF is utilised for the adsorbed phase. The proposed
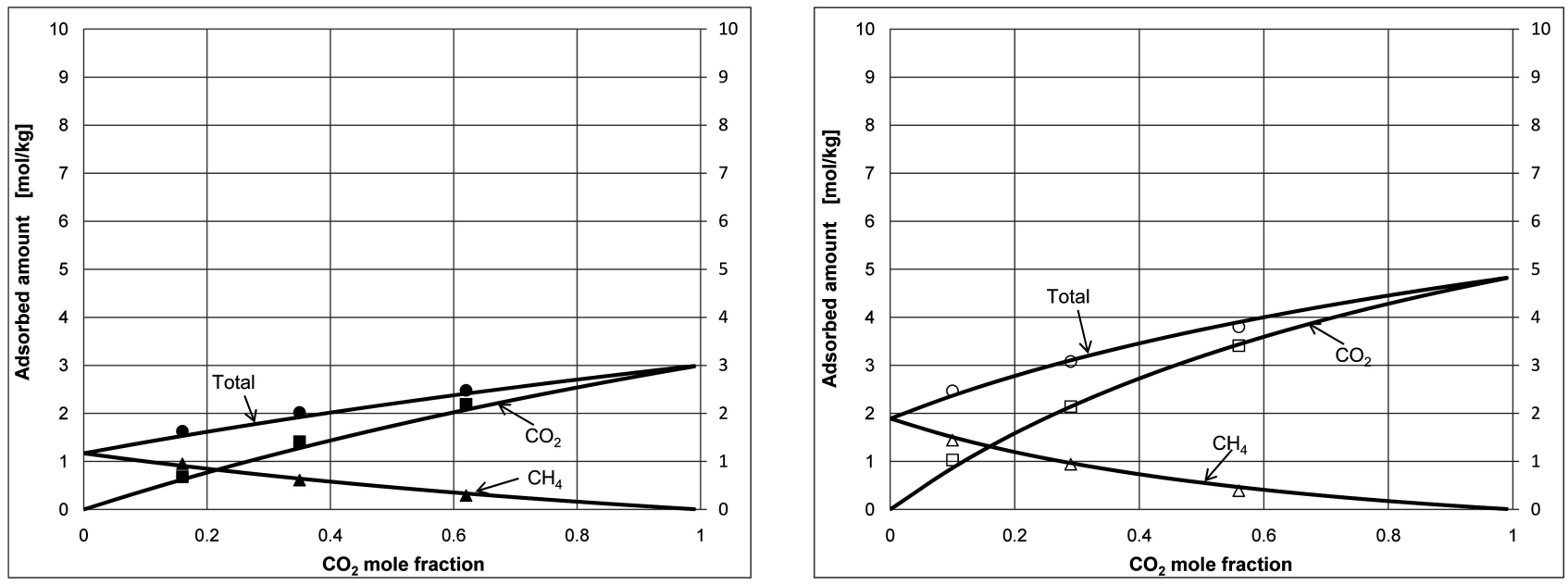

Fig. (2). Co-adsorption isotherms on activated carbon for the binary mixture methane/carbon dioxide at $0.1 \mathrm{MPa}$. The experimental data taken from Ref. [10] are represented by solid symbols at sf:p01t298: $298 \mathrm{~K}$ and by open symbols at sf:p01t273: 273 K. Triangles, squares and circles are used for $\mathrm{CH}_{4}, \mathrm{CO}_{2}$ and total, respectively. Solid lines represent corresponding values calculated by the model. See also Fig. (6) in Ref. [10].
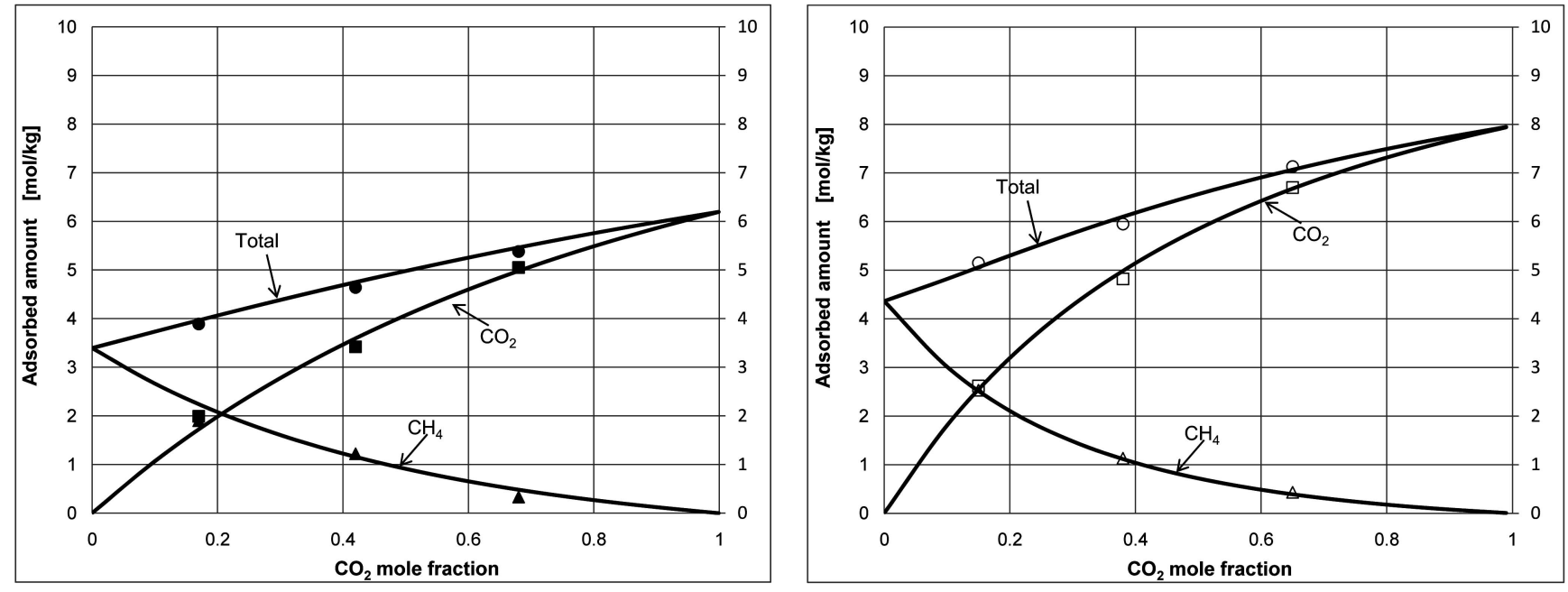

Fig. (3). Co-adsorption isotherms on activated carbon for the binary mixture methane/carbon dioxide at $0.5 \mathrm{MPa}$. The experimental data taken from Ref. [10] are represented by solid symbols at sf:p05t298: $298 \mathrm{~K}$ and by open symbols at sf:p05t273: $273 \mathrm{~K}$. Triangles, squares and circles are used for $\mathrm{CH}_{4}, \mathrm{CO}_{2}$ and total, respectively. Solid lines represent corresponding values calculated by the model. See also Fig. (3) in Ref. [10]. 

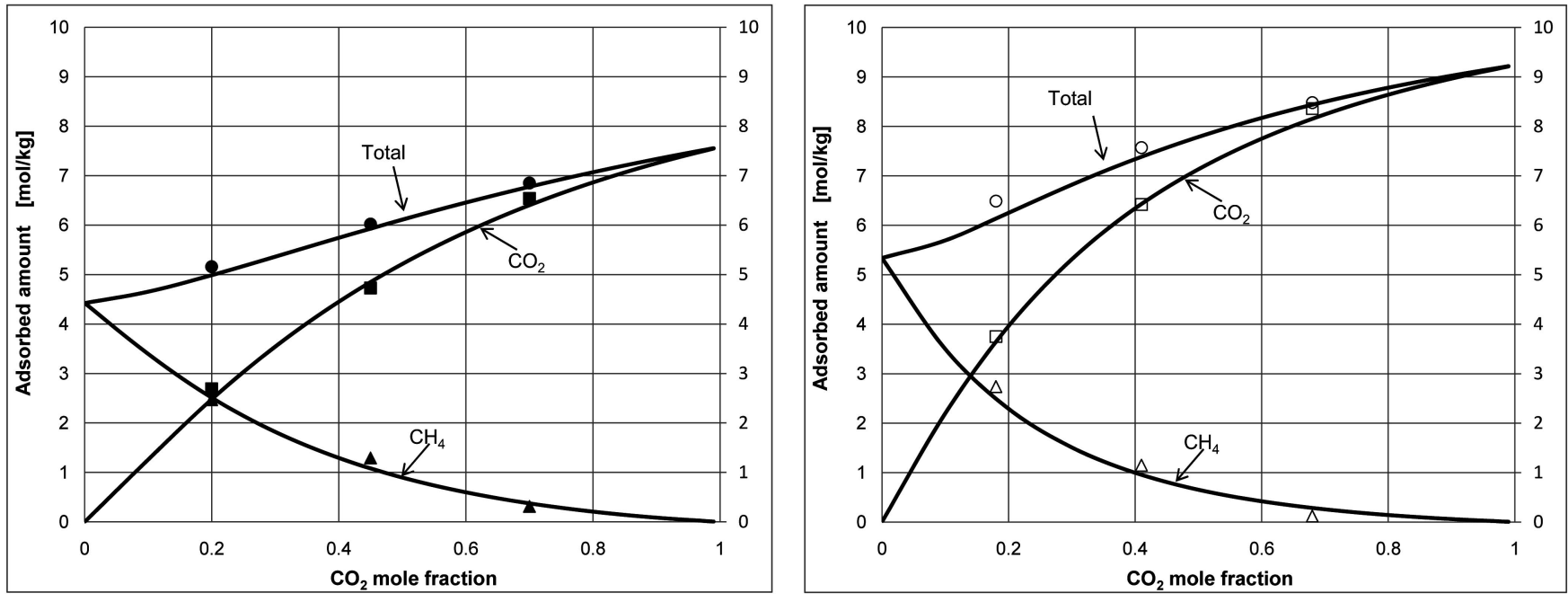

Fig. (4). Co-adsorption isotherms on activated carbon for the binary mixture methane/carbon dioxide at $1 \mathrm{MPa}$. The experimental data taken from Ref. [10] are represented by solid symbols at sf:p1t298: $298 \mathrm{~K}$ and by open symbols at sf:p1t273: $273 \mathrm{~K}$. Triangles, squares and circles are used for $\mathrm{CH}_{4}, \mathrm{CO}_{2}$ and total, respectively. Solid lines represent corresponding values calculated by the model. See also Fig. (7) in Ref. [10].

model allows predicting the adsorption behaviour of $\mathrm{CO}_{2} /$ $\mathrm{CH}_{4}$ mixtures on coal, which is of particular importance for modelling $\mathrm{CO}_{2}$ sequestration and enhanced CBM recovery.

\section{REFERENCES}

[1] E. P. Robertson, "Enhanced coal bed methane recovery and $\mathrm{CO}_{2}$ sequestration in the Powder river basin," Idaho National Laboratory, Technical Report, June 2010. [Online]. Available: http://www.inl.gov/technicalpublications/Documents/4536713.pdf . Koter, and A. P. Terzyk, "Two-dimensional gas and vacancy solution approaches in the thermodynamic description of adsorption equilibrium," Journal of Colloid and Interface Science, vol. 282, pp. 335-339, 2005.

[3] A. Myers, "Thermodynamics of adsorption in porous materials," AIChE Journal, vol. 48, no. 1, pp. 145-160, 2002.

[4] B. P. Bering, M. M. Dubinin, and V. V. Serpinsky, "On thermodynamics of adsorption in micropores," Journal of Colloid and Interface Science, vol. 38, no. 1, pp. 185-194, 1972.

[5] B. P. Bering, and V. V. Serpinskii, "Adsorption equilibrium and thermodynamics of vacancy solutions," Russian Chemical Bulletin, vol. 22 , no. 12 , pp. 2616-2621, 1973.

[6] D. N. Saulov, I. G. Vladimirov, and A. Klimenko, "Modified associate formalism without entropy paradox: Part I: model description," Journal of Alloys and Compounds, vol. 473, pp. 167$175,2009$.
[7] D. N. Saulov, and A. Klimenko, "Modified associate formalism without entropy paradox: Part II: comparison with similar models," Journal of Alloys and Compounds, vol. 473, pp. 157-162, 2009.

[8] L. P. Ding, and S. K. Bhatia, "Application of heterogeneous vacancy solution theory to characterization of microporous solids," Carbon, vol. 39, pp. 2215-2229, 2001.

[9] S. K. Bhatia, and L. P. Ding, "Vacancy solution theory of adsorption revisited," AIChE Journal, vol. 47, no. 9, pp. 2136$2138,2001$.

[10] V. Goetz, O. Pupier, and A. Guillot, "Carbon dioxide-methane mixture adsorption on activated carbon," Adsorption, vol. 12, no. 1, pp. 55-63, 2006.

[11] M. M. Dubinin, "Physical adsorption of gases and vapors in micropores," in Progress in Surface and Membrane Science, D. A. Cadenhead, J. E. Danielli, and M. D. Rosenberg, Eds. $1 \mathrm{em}$ plus $0.5 \mathrm{em}$ minus $0.4 \mathrm{em}$ Academic Press, New-York, 1975, p. $1 \hat{\mathrm{A}}-70$.

[12] A. Lavanchy, M. StÃ $\llbracket c k l i$, C. Wirz, and F. Stoeckli, "Binary adsorption of vapours in active carbons described by the Dubinin equation," Adsorption Science and Technology, vol. 13, no. 6, pp. 537-545, 1996.

[13] A. L. Myers and J. M. Prausnitz, "Thermodynamics of mixed-gas adsorption," AIChE Journal, vol. 11, no. 1, pp. 121-127, 1965.

[14] I. Barin, "Therrnochernical Data of Pure Substances", 3rd ed., K. Sora and J. Gardiner, Eds. 1em plus $0.5 \mathrm{em}$ minus $0.4 \mathrm{em}$ VCH Publishers, Inc., New York, 1995, (in collaboration with Gregor Platzki). 